LA-UR-

Title:

Author(s):

Submitted to:

\section{PHYSICS-BASED GENERATION OF GAMMA-RAY RESPONSE FUNCTIONS FOR CDZNTE DETECTORS}

T. H. Prettyman, D. J. Mercer, C. S. Cooper, P. A. Russo, M. Rawool-Sullivan, D. A. Close, P. N. Luke, M. Amman, and S. Soldner

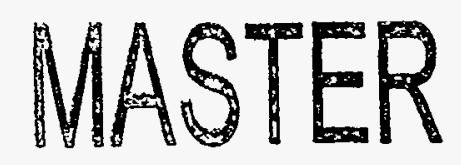

DISTRIBUTION OF THIS DOCUMENT IS UNLIMITED

International Topical Conference on Methods and Applications of Radioanalytical Chemistry - MARC IV Kona, Hawaii April 6-11, 1997 (FULL PAPER)

\title{
DISCLAIMER
}

This report was prepared as an account of work sponsored by an agency of the United States Government. Neither the United States Government nor any agency thereof, nor any of their employees, makes any warranty, express or implied, or assumes any legal liability or responsibility for the accuracy, completeness, or usefulness of any information, apparatus, product, or process disclosed, or represents that its use would not infringe privately owned rights. Reference herein to any specific commercial product, process, or service by trade name, trademark. manufacturer, or otherwise does not necessarily constitute or imply its endorsement, recommendation, or favoring by the United States Government or any agency thereof. The views and opinions of authors expressed herein do not necessarily state or reflect those of the United States Government or any agency thereof.
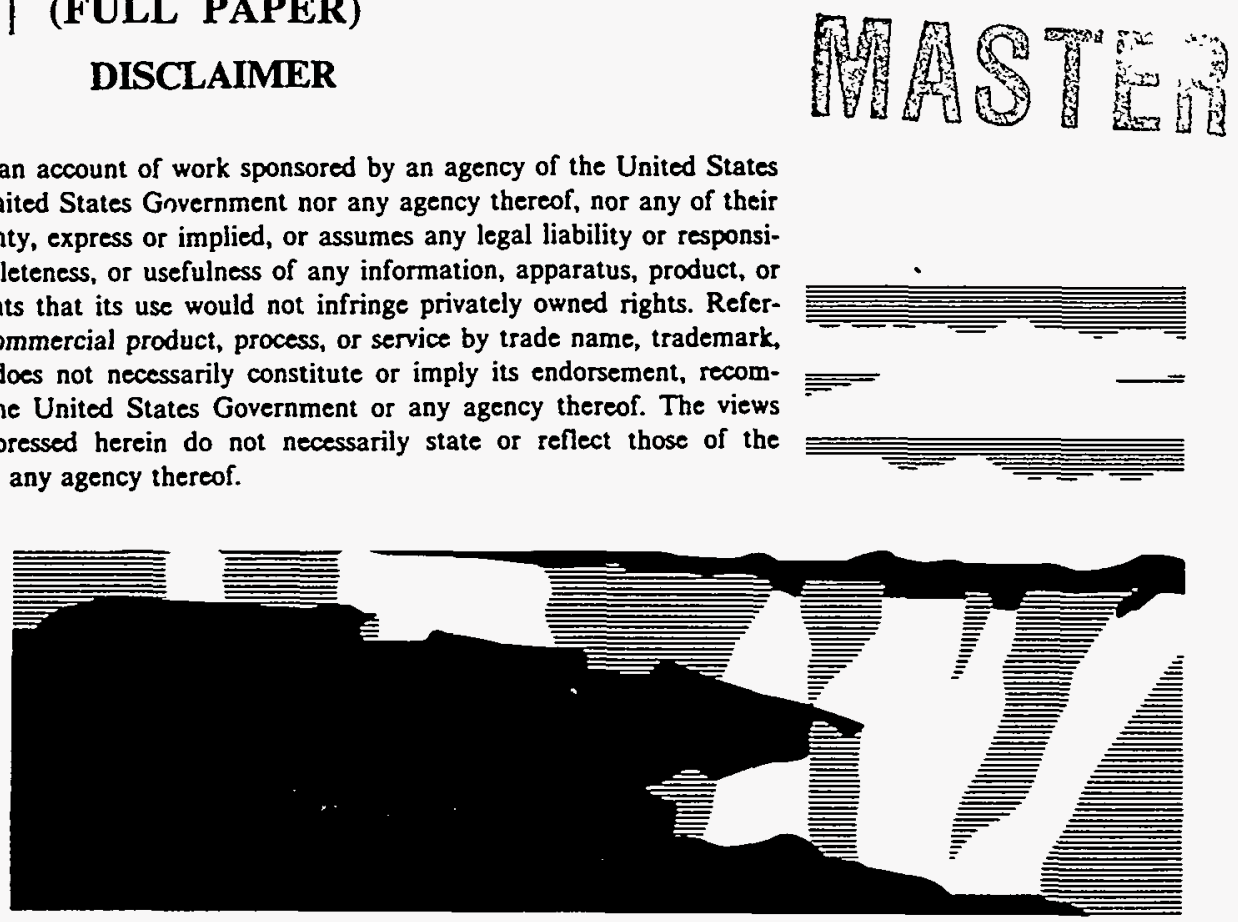

Los Alamos National Laboratory, an affirmative action/equal opportunity emplofyer, is operated by the University of California for the U.S. Department of Energy under contract W-7405-ENG-36. By acceptance of this article, the publisher recognizes that the U.S. Government retains a nonexclusive, royalty-free license to publish or reproduce the published form of this contribution, or to allow others to do so, for U.S. Govemment purposes. The Los Alamos National Laboratory requests that the publisher identify this article as work performed under the auspices of the U.S. Department of Energy. 


\section{DISCLAIMER}

Portions of this document may be illegible in electronic image products. Images are produced from the best available original document. 


\section{DRAFT}

\section{PHYSICS-BASED GENERATION OF GAMMA-RAY RESPONSE FUNCTIONS FOR CdZnTe}

DETECTORS

T. H. Prettyman, D. J. Mercer, C. Cooper, and P. A. Russo, M. Rawool-Sullivan, and D. A. Close Los Alamos National Laboratory

P. N. Luke and M. Amman, Lawrence Berkeley National Laboratory

S. Soldner, eV Products

\section{Abstract}

A physics-based approach to gamma-ray response-function generation is presented in which the response of $\mathrm{CdZnTe}$ detectors is modeled from first principles. Computer modeling is used to generate response functions needed for spectrum analysis for general detector configurations (e.g., electrode design, detector materials and geometry, and operating conditions). With computer modeling, requirements for calibration and characterization are significantly reduced. Elements of the physics-based model, including gamma-ray transport, charge drift-diffusion, and circuit response, are presented. Calculated and experimental gamma-ray spectra are compared for a coplanar-grid CdZnTe detector.

\section{INTRODUCTION}

Improvements in material quality and detector design have led to the development of large-volume $\mathrm{CdZnTe}$ detectors $\left(\sim 2 \mathrm{~cm}^{3}\right)$ that function effectively as gamma-ray spectrometers. CdZnTe is a wide-bandgap, compound semiconductor that is used routinely in room-temperature detectors. Spectrometer-grade $\mathrm{Cd}_{\mathrm{x}} \mathrm{Zn}_{1-\mathrm{x}} \mathrm{Te}$ material is grown commercially using the high-pressure Bridgeman process (Parnham, 1996). Large samples have been manufactured that have high bulk resistivity, low surface-leakage current, and relatively uniform electronic properties. The availability of such samples is presently low; however, mass production of spectrometer-grade material could be realized in the next few years.

Prior to 1994, the size of CdZnTe detectors for gamma-ray spectroscopy was limited by poor hole mobility. This limitation has been overcome in large measure by the development of the coplanar-grid technique (Luke, 1994). Coplanar grids perform the same function as Frisch-grids used in gas proportional counters (Frisch, 1944). The effect of current induced by charge motion within the volume of the detector is 


\section{DRAFT}

essentially eliminated. Charge motion is sensed only near the anode of the device. Consequently, the response is dominated by electrons. Because the e-folding distance for electron trapping is relatively large (up to $5 \mathrm{~cm}$ at $1000 \mathrm{~V} / \mathrm{cm}$ ), large detectors can be constructed with excellent resolution and peak shape. The improvement that can be achieved by the application of coplanar grid detectors is illustrated in Figure 1 for a $0.5 \mathrm{~cm}^{3}$ detector manufactured by $\mathrm{eV}$ Products. The resolution of large-volume $\left(>2 \mathrm{~cm}^{3}\right)$ detectors with coplanar grids is highly variable, but is typically better than $5 \%$ FWHM at $662 \mathrm{keV}$. The resolution and stability of coplanar-grid detectors are significantly better than $\mathrm{NaI}(\mathrm{Tl})$ detectors with photomultiplier tubes.

The development of coplanar grids has lead to renewed interest in CdZnTe as a substitute for HPGeand $\mathrm{NaI}(\mathrm{Tl})$-detectors for many applications, particularly those where detector size, ruggedness, and maintenance needs are limiting factors. For example, a number of nuclear nonproliferation activities could benefit from the use of CdZnTe. Burgeoning applications include detection of nuclear-material smuggling, materials control and accountability, search, and inspection. Most of these applications require portable instrumentation that is capable of identifying and quantifying material with complex gamma-ray spectra (e.g., variable-burnup plutonium). With the improvement in resolution and efficiency enabled by coplanar-grids, $\mathrm{CdZnTe}$ detectors are expected to replace $\mathrm{NaI}(\mathrm{Tl})$ detectors for portable measurements.

Because a wide-range of applications is anticipated, a general capability for analyzing CdZnTe gammaray spectra is desired. Accurate response functions for fitting gamma-ray spectra are needed for different detector designs and different operating conditions. For established detector technology, particularly HPGe, a semi-empirical approach is used to model response functions. The spectrum is decomposed into basic features, some of which are derived empirically. For example, HPGe line shapes can be modeled as the combination of a Gaussian to represent the full-energy peak, exponential functions for tailing below the peak, and a flat continuum (Jin, 1986). This approach works because the basic shapes are reproduced for most detectors considered. A few experiments are required to determine the parameters of functions used to represent the response. 


\section{DRAFT}

- The response of CdZnTe detectors depends on a number of factors that can vary considerably between detectors, including material electronic properties, detector geometry, electrode and circuit design, and packaging. Bulk electronic quantities, particularly the mobility-lifetime product for holes, can vary significantly between detectors. In addition, the uniformity of the material can influence performance for spectroscopy (Van Scyoc, 1996; Luke, June 1996). The magnitude of surface effects, such as leakage current and trapped charge, is also variable and depends on manufacturing processes and passivation methods. The response is also influenced by electrode design. Various designs have been implemented for coplanar-grid detectors, each producing a different characteristic peak shape. Peak shape is also influenced by the operating conditions (e.g. applied bias, difference-circuit gain, and differential bias). The response of $\mathrm{CdZnTe}$ detectors can be sensitive to the distribution of gamma-ray interaction sites within the detector. Consequently, the design of the detector collimator and packaging also influence the response.

Because the performance of $\mathrm{CdZnTe}$ detectors is variable, a single set of empirical or semi-empirical response functions is inadequate. In effect, basic response components must be "discovered" for each detector or class of detectors (e.g. planar vs. coplanar-grid vs. coaxial). Consequently, detector characterization can be time-consuming and costly. Semi-empirical models do not have the flexibility needed to cover the range of applications and operating conditions envisioned for these devices.

To provide a more general capability for response function characterization, we are developing a physically-based model of semiconductor detectors. The basic mechanisms underlying the detector response are modeled from first principles. The transport of gamma-rays from the source to the detector is simulated using the Monte Carlo method, which allows precise modeling of the detector collimator and packaging, and, if needed, the geometry and composition of the sample and intervening materials. The calculation includes the production of charge carriers within the detector for discrete gamma-ray interactions and the distribution of carriers caused by secondary transport effects (x-ray and electron transport). A separate calculation is used to model charge-carrier motion and collection, and electrode currents. The electronic properties of the material and the geometry of the electrodes are modeled. Spatial maps of the charge-induction efficiency are combined 


\section{DRAFT}

with the Monte Carlo simulation to generate pulse-height spectra. A computationally efficient method to map the spatial response of the detector to charge motion for 2- or 3-dimensional devices has been developed.

The physics-based model can be applied to generate response functions for a wide-variety of detector designs and operating conditions. In principle, accurate response functions can be generated rapidly with relatively little experimental characterization if the design of the detector and operating conditions are known.

In this paper, an overview of the modeling techniques is presented. The model is applied to generate response functions for a coplanar-grid CdZnTe detector. The merits and limitations of the physics-based approach are discussed.

\section{THEORY AND METHODS OF COMPUTATION}

In order to develop a complete model of semiconductor detectors, the following physical processes must be treated:

- transport of gamma-rays from the source to the detector,

- production of charge carriers (electron-hole pairs) in the detector by gamma-ray events,

- transport of charge in the detector by drift and diffusion and collection of charge at the contacts,

- electrode currents (displacement and conduction) and the response of circuit components,

- noise associated with carrier production and trapping, leakage current, and electronics.

The Monte Carlo method is used to model gamma-ray transport. For research purposes, a set of subroutine modules that model specific aspects of gamma-ray transport are used (e.g., sampling direction cosines for incoherent scattering). A general, three-dimensional geometry package is used to simulate the detector and surroundings, including collimators, packaging and electronics, and samples. The modular approach was selected to allow complete control over the design of tallies and variance reduction methods for specific applications. 


\section{DRAFT}

- The objective of the Monte Carlo gamma-ray transport calculation is to estimate the production of charge carriers in the detector for each gamma-ray event. The number of electron-hole pairs is assumed to be proportional to the energy deposited by the gamma-ray. The energy required to create an electron-hole pair varies with the molar fraction of $\mathrm{Zn} .(-5 \mathrm{eV}$ for $\mathrm{x}=0.1)$. The distribution of electron-hole pairs is to first order given by the location of the interaction sites. Secondary transport effects, however, can be important for some devices. For example, most photoelectric interactions in $\mathrm{CdZnTe}$ result in the production the $\mathrm{Cd} \mathrm{K} \alpha 1 \mathrm{x}$-ray which has a mean-free-path of $130 \mu \mathrm{m}$. The range of Compton electrons and photoelectrons with energies above $200 \mathrm{keV}$ is greater than $100 \mu \mathrm{m}$. Because significant variations in the collection efficiency can occur on this scale, particularly in coplanar-grid detectors, secondary transport effects must be modeled.

The transport of charge-carriers is simulated using a "small-transient" model in which it is assumed that the charge deposited by a gamma-ray does not perturb the steady-state solution for the device. The steadystate electric field can be determined by solving Poisson's equation:

$-\nabla \cdot(\varepsilon \nabla \varphi)=\rho / \varepsilon_{0}$

Eq. 1

where $\varphi$ is electrostatic potential, $\varepsilon$ is the dielectric constant, and $\rho$ is the space charge density. Using this approach, contacts with fixed potential can be modeled. Von Neumann boundary conditions are used to model trapped surface charge and insulated surfaces. A finite-elements code is used to calculate the potential for general two-dimensional devices.

The motion of charge in the device is determined by solving the carrier continuity equations. For example, the continuity equation for electrons is given by

$$
\frac{d n}{d t}=-\nabla \cdot\left(n \mu_{n} \nabla \varphi\right)+\nabla \cdot\left(D_{n} \nabla n\right)-n / \tau_{n}
$$

where $n$ is the number density of electrons, $\mu_{n}$ is the mobility, $D_{n}$ is the diffusion coefficient, and $\tau_{n}$ is electron lifetime. A similar equation is solved separately for the hole concentration, $p$. For spectrometer-grade material, the diffusion coefficient for electrons is on the order of $30 \mathrm{~cm}^{2} / \mathrm{s}$ (by the Einstein relation). 


\section{DRAFT}

Dispersion of electrons by diffusion can be important in large devices. For example, under nominal operating conditions $(1000 \mathrm{~V} / \mathrm{cm}$ ) the dispersion of electrons (width of the charge cloud) can approach $250 \mu \mathrm{m}$ in devices that are $1 \mathrm{~cm}$ thick. The maximum rise-time under these conditions is $\sim 1 \mu \mathrm{s}$. If diffusion is neglected, the continuity equation can be represented by a set of coupled, ordinary differential equations.

The current induced in electrodes by the motion of charge in the device is modeled using Ramo's theorem (Ramo, 1939; Beck, 1953; Cavalleri, 1963). The response of individual electrodes or groups of electrodes connected by a common circuit can be modeled. For example, the current induced in a circuit (denoted $k$ ) by electrons is given by

$\frac{d Q_{k}^{n}}{d t}=q \int_{\mathbf{v}} d^{3} r n \mathbf{v} \cdot \nabla \varphi_{k} \quad Q_{k}^{n}(0)=0$

where $q$ is the electron charge, $\mathbf{v}$ is the velocity of the electrons, and $\varphi_{K}$ is the weighting potential given by the solution of Laplace's equation:

$$
\nabla \cdot\left(\varepsilon \nabla \varphi_{k}\right)=0
$$

The electrodes associated with circuit $k$ are set to unit potential. All other electrodes are grounded. The induced charge, $Q_{k}(t)=Q_{k}^{n}(t)+Q_{k}^{p}(t)$, can be used to approximate the output of a charge-sensitive preamplifier and is the starting point for more detailed calculations of circuit response.

In order to simulate pulse-height spectra, the charge induction efficiency must be determined for each point in the detector. Charge induction efficiency $(\eta)$ is defined as the ratio of the preamplifier output after the charge is collected to the charge initially deposited at the interaction site. The induction efficiency at any point within the detector can be estimated by solving the small-transient model (Eqs. 1-4) for an impulse of charge at the selected location. For example, the efficiency of circuit $k$ for induction by electrons at $\mathbf{r}$ is given by $\eta_{k}^{n}(\mathbf{r})=\lim _{t \rightarrow \infty} Q_{k}^{n}(t) / q$ if the initial condition is an impulse of electrons, $n(\mathbf{r}, 0)=\delta\left(\mathbf{r}^{\prime}-\mathbf{r}\right)$. The pulse-height for an event can be calculated from the induction efficiency and is given by 


\section{DRAFT}

$H_{k} \propto \sum_{i=1}^{l} \int_{V} d^{3} r\left[\eta_{k}^{n}(\mathbf{r})+\eta_{k}^{p}(\mathbf{r})\right] N_{i}(\mathbf{r})$

Eq. 5

where $I$ is the number of discrete interactions in the detector and $N_{i}(\mathbf{r})$ is the number density of electron-hole pairs for each interaction.

There are two general strategies for mapping induction efficiency: forward and reverse mapping. Forward mapping involves the direct solution of the semiconductor equations starting at each point in the detector volume. This approach is simple, but time consuming, particularly for gridded or pixellated detectors for which the induction efficiency has high-frequency spatial components. For most detectors, forward mapping is impractical. The alternative is reverse mapping, in which charge carriers start from the contacts and drift backwards through the detector. Reverse mapping is a novel approach developed by Los Alamos that involves the solution of an adjoint semiconductor model (Prettyman,1997). The induction efficiency can be determined by solving the adjoint equations with the appropriate initial and boundary conditions. It should be emphasized that with this approach, a single transient calculation is sufficient to determine the induction efficiency at every point in the device. This enables the rapid variation of device parameters needed for response function analysis and generation.

\section{RESPONSE FUNCTIONS FOR A COPLANAR-GRID DETECTOR}

To demonstrate the physics-based approach, we modeled response functions for a $1 \mathrm{~cm} \times 1 \mathrm{~cm} \times 0.5$ $\mathrm{cm}$, coplanar-grid detector manufactured by $\mathrm{eV}$ Products. A diagram of the device is shown in Figure 2. The anode consists of 16 inter-digital strips connected to separate preamplifier circuits ( $A$ and $B$ ). The strips are $250 \mu \mathrm{m}$ wide. The cathode is a single, full-area electrode. Negative bias (typically $-600 \mathrm{~V}$ ) is applied to the cathode through a high-voltage filter to produce a relatively uniform electric field (in excess of $1000 \mathrm{~V} / \mathrm{cm}$ ) throughout the volume of the detector. A small differential bias is applied to the anode so that the strips connected to circuit A have higher potential than those connected to circuit B. Circuit B is grounded. With sufficient differential bias, electrons are collected exclusively by circuit $\mathrm{A}$. 


\section{DRAFT}

- The current induced by the motion of charge in the detector is the similar for circuits $A$ and $B$ except for a narrow region near the anode. So, by subtracting the signal for circuit $B$ from circuit $A$, the effect of charge motion within the detector volume is eliminated. This is accomplished by a difference circuit. The response of this circuit is dominated by electron motion and, since the mobility-lifetime of electrons is relatively large, the response of the difference circuit is insensitive to the origin of the charge. To demonstrate this effect, the induction efficiency of the difference circuit is shown as a function of depth (distance from the anode) in Figure 2. Uniform induction efficiency is achieved by adjusting the gain of circuit B prior to subtraction to compensate for electron trapping.

The electric potential and the weighting potential (for circuit A) used to calculate the induction efficiency are shown in Figure 3. The device was modeled in two-dimensions. The surrounding air and container were modeled to account for fringe effects. Following Nemirovsky (1996), the dielectric constant of $\mathrm{CdZnTe}$ was assumed to be 11 . Because the electrodes are not centered on the face of the detector, the weighting potential for circuit A varies across the detector (from left to right). The weighting potential for the $\mathrm{B}$ circuit is the mirror image of $\mathrm{A}$. Because the weighting potential for circuits $\mathrm{A}$ and $\mathrm{B}$ are different, charge motion near the edge of the detector is not accurately compensated for by the difference circuit. This effect is illustrated in Figure 4 for calculated and experimental difference-circuit pulses corresponding to different regions of the detector. Edge effects can cause significant degradation in peak shape. Recent. grid designs have incorporated features that minimize edge effects (Luke, November 1996).

Induction efficiency maps computed using an adjoint mapping algorithm with the drift approximation are displayed in Figure 5 for each charge carrier and for both circuits. The maps were computed for a differential bias of $30 \mathrm{~V}$. All of the maps exhibit a lateral variation that can be attributed to the weighting potential. The most striking features, however, are grooves in the electron induction maps that correspond to events for which the electrons were collected by the opposite circuit. The width and depth of the grooves depends on the differential bias. With large differential bias ( $>60 \mathrm{~V})$, the detector is fully depleted (all of the 


\section{DRAFT}

charge is collected by circuit A); however, local variations in electron induction efficiency persist due to differences in the paths that electrons follow near the electrodes.

Variations in induction efficiency due to charge transport have a pronounced effect on peak shape, particularly low-energy tailing, which can be observed by adjusting differential bias. The differential bias for which depletion occurs, however, is not accurately predicted by the calculation. Experiments show that depletion occurs for most detectors at very low differential bias $(<10 \mathrm{~V})$. Several explanations for the difference between the calculation and the experiments have been proposed. For example, the discrepancy is consistent with fixed surface charge (e.g., between the electrodes). Experiments are being planned to help better understand this effect.

To test the validity of the model, we measured pulse-height spectra for several isotopes. The conditions of the experiment, including the geometry of the detector and surrounding materials, were carefully controlled. A complete geometric model of the apparatus was developed. Pulse-height spectra were computed by Monte Carlo. The majority of parameters used to generate the induction maps used in the calculation were either known (e.g., grid design) or measured (e.g., mobility-lifetime products). Carrier mobilities were taken from the literature. The mobility of holes was assumed to be $50 \mathrm{~cm}^{2} / \mathrm{V}$-s. The mobility of electrons was assumed to be $800 \mathrm{~cm}^{2} / \mathrm{V}$-s. The magnitude of the electronic noise used in the calculation was determined experimentally. Noise due to fluctuations in the charge-carrier population was assumed to be negligible compared to the electronic component (Niemela, 1994).

A comparison between calculated and experimental pulse-height spectra is shown in Figure 6 for lowenergy (Co-57, $122 \mathrm{keV})$ and high-energy (Cs-137, $662 \mathrm{keV})$ gamma-ray sources. All of the spectral features that appear in the experiment are reproduced by the calculation. Calculated and experimental metrics (e.g., peak FWHM and FWTM, peak-to-Compton ratio, and peak-to-valley ratio) agree to within a few percent. To test the ability of the model to predict relative efficiency, a pulse-height spectrum was measured for a source with widely spaced gamma-rays (Se-75). The comparison of calculation to experiment in Figure 7 shows that the model accurately predicts efficiency over a wide range of energies. Using the model, a response function 


\section{DRAFT}

library was generated for gamma-rays ranging from $100 \mathrm{keV}$ to $700 \mathrm{keV}$ in $50 \mathrm{keV}$ increments (Figure 8). The library is suitable for the quantitative analysis of complex spectra.

\section{SUMMARY AND CONCLUSIONS}

A comprehensive, physics-based model of CdZnTe detectors has been demonstrated. Physics-based modeling provides detailed information needed to investigate basic mechanisms underlying detector response. Calculations of the response can be carried out rapidly. An adjoint method has been developed that, in principle, enables the charge induction efficiency for the entire detector to be mapped with a single transient calculation. In addition, because the transport of gamma-rays is independent of the semiconductor model, pulse-height spectra can be tallied in the Monte Carlo calculation for a large number of induction maps simultaneously with essentially no throughput penalty. Consequently, the effect of variations in a large number of parameters can be determined quickly. This capability is essential for determining response function parameters.

The accuracy of response-functions obtained with the physics-based model for the coplanar grid detector is sufficient for quantitative spectrum analysis. Potential benefits of the physics based approach include: reduced effort for characterization (fewer experiments are required to characterized detector response since most of the parameters are known or can be measured independently); simplicity (the same model is used for all detectors); and flexibility (the model can be used to generate response functions for a wide range of measurement conditions). The model is sufficiently general that it can be applied to simulate most device structures currently considered for $\mathrm{CdZnTe}$ radiation detectors. Work is currently underway to extend the semiconductor model to three-dimensions so that pixel structures for imaging detectors can be modeled. More effort is required to understand surface and contact effects in CdZnTe and their influence on charge carrier transport. A better understanding of these effects would further reduce the amount of effort needed for detector characterization. 
ACKNOWLEDGEMENT

\section{DRAFT}

This work was supported by the Department of Energy through a Laboratory Directed Research and

Development, Program Development Project.

\section{REFERENCES}

A. H. W. Beck, Thermionic Valves: Their Theory and Design, Cambridge at the University Press, 1953.

Cavalleri, G., et al. (1963), "On the Induced Charge in Semiconductor Detectors," Nucl. Instr. Meth., Vol. 21, pp. 177-178.

O. R. Frisch (1944), "Isotope Analysis of Uranium Samples by Means of their $\alpha$-Ray Groups," British Atomic Energy Agency report BR-49.

Jin, Y., R. P. Gardner, and K. Verghese (1986), "A Semi-Emperical Model for the Gamma-Ray Response Function of Germanium Detectors Based on Fundamental Interaction Mechanisms," Nucl. Instr. Meth. Phys. Res., Vol. A242, pp. 416-426.

Luke, P. N. (November 28, 1994) "Single-Polarity Charge Sensing in Ionization Detectors Using Coplanar Electrodes", Appl. Phys. Lett., Vol. 65, pp. 2884-2887.

Luke, P. N. and E. E. Eissler (June, 1996), "Performance of CdZnTe Coplanar-Grid Gamma-Ray Detectors," IEEE Transactions on Nuclear Science, Vol. 43, No. 3, pp. 1481-1486.

Luke, P. N., et al. (November, 1996), "Electrode Design for Coplanar-Grid Detectors," Presented at the IEEE Nuclear Science Symposium, November 3-9, Anaheim, Ca, submitted to IEEE Transactions on Nuclear Science, to appear.

Nemirovsky, Y., et al. (1996), "Study of the Charge Collection Efficiency of CdZnTe Radiation Detectors," Journal of Electronic Materials, Vol. 25, No. 8, pp. 1221-1231.

Niemela, A. and H. Sipila (1994), "Evaluation of CdZnTe Detectors for Soft X-Ray Applications," IEEE Transactions on Nuclear Science, Vol. 41 (No. 4), pp. 1054-1057.

K. B. Parnham (1996), "Recent Progress in $\mathrm{Cd}_{1-\mathrm{x}} \mathrm{Zn}_{\mathrm{x}} \mathrm{Te}$ Radiation Detectors," Nucl. Instr. Meth. Phys. Res., Vol. A377, pp. 487-491.

T. H. Prettyman (1997), "An Adjoint Method for Mapping Charge Induction Efficiency in Semiconductor Detectors," Informal report (to be published).

Ramo, S. (September, 1939), “Currents Induced by Electron Motion,” P.I.R.E., Vol. 27, pp. 584-585.

Van Scyoc, J. M., et al. (1996), "Material Inhomogeneities in $\mathrm{Cd}_{1-\mathrm{x}} \mathrm{Zn}_{\mathrm{x}} \mathrm{Te}$ and Their Effects on Large Volume Gamma-Ray Detectors," Journal of Electronic Materials, Vol. 25, No. 8, pp. 1323-1327. 


\section{DRAFT}

\section{FIGURES}

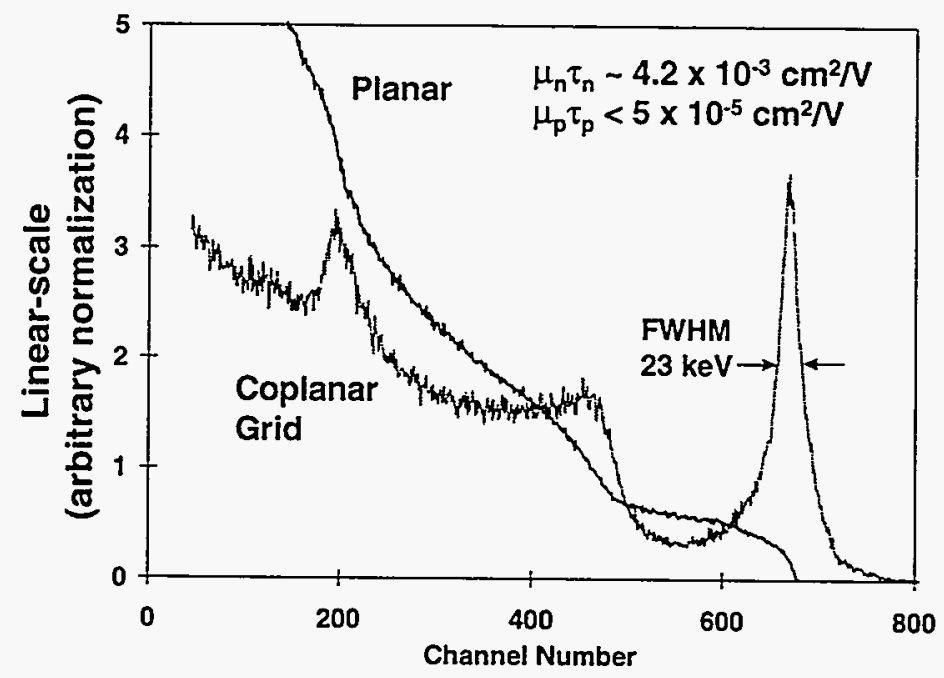

Figure 1. Comparison between planar and coplanar-grid spectra.for a $1 \mathrm{~cm} \mathrm{x} 1 \mathrm{~cm} \times 0.5 \mathrm{~cm} \mathrm{CdZnTe}$ detector.

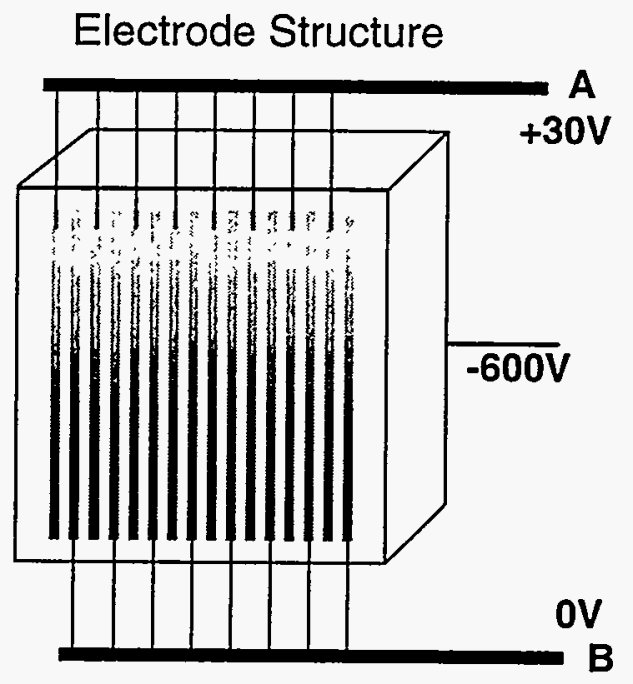

Charge Induction Efficiency

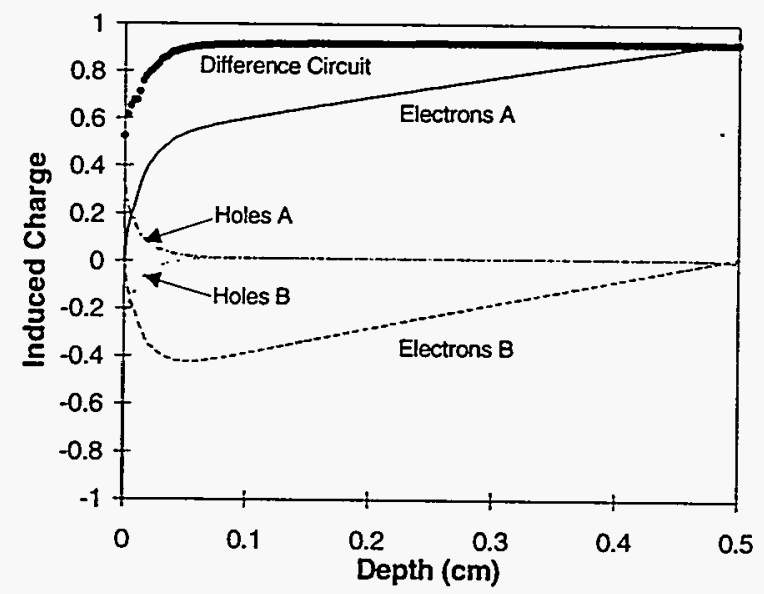

Figure 2. Electrode design and operating parameters for the $0.5 \mathrm{~cm}^{3}$ coplanar-grid detector (left). Charge induction efficiency as a function of depth (right). Mobility-lifetime products used in the calculation are shown in Figure 1. 


\section{DRAFT}
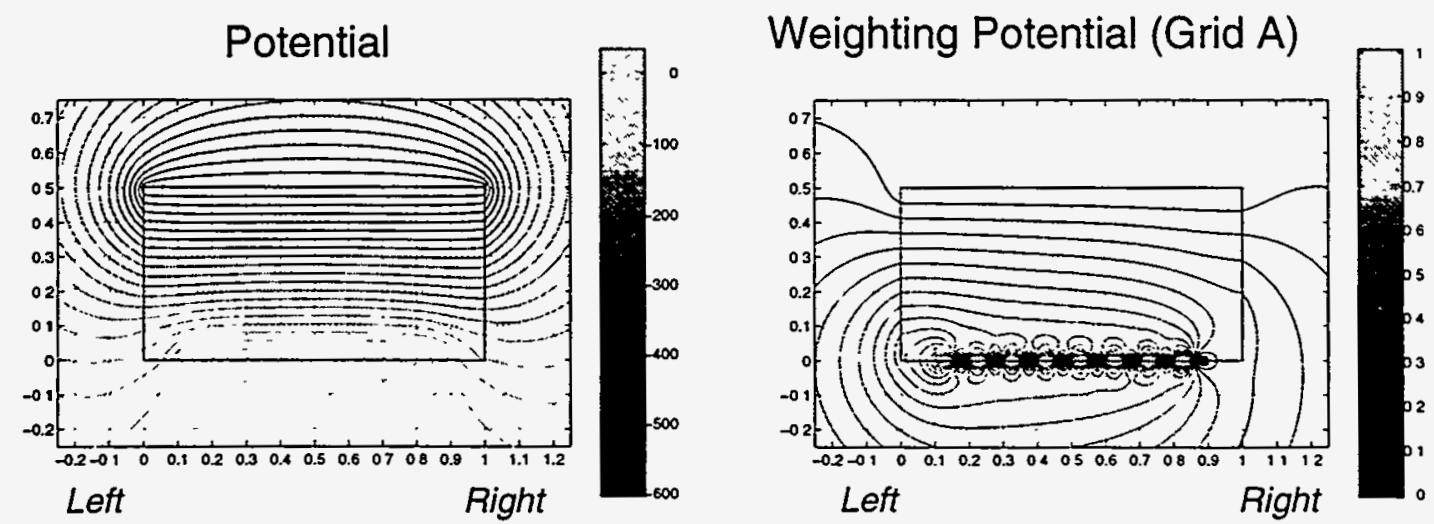

Figure 3. Electrostatic potential (left) and the weighting potential for electrodes connected to circuit A of the coplanar grid detector (right).

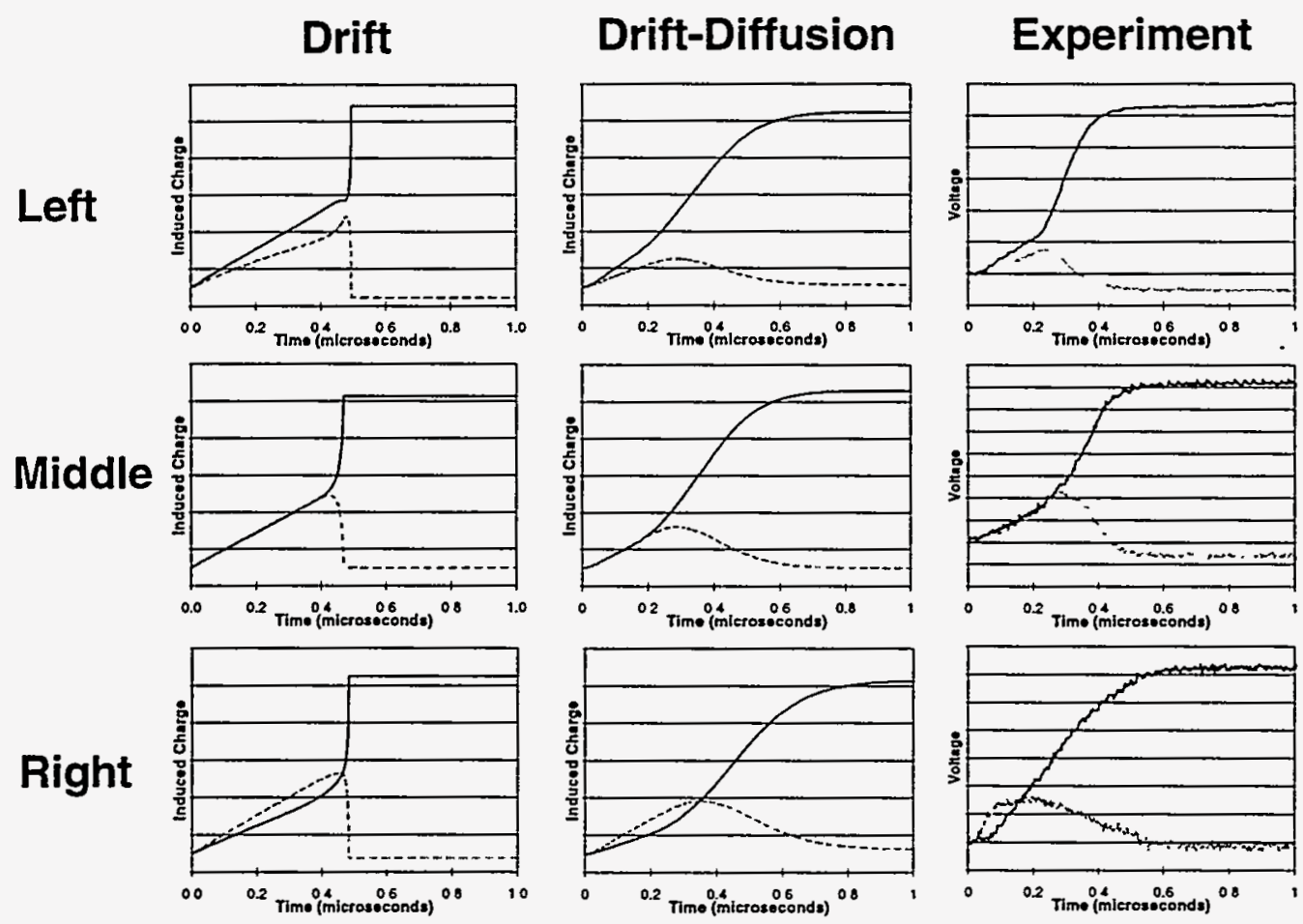

Figure 4. Comparison between calculated and experimental pulse-shapes for events in different regions of the detector. 


\section{DRAFT}
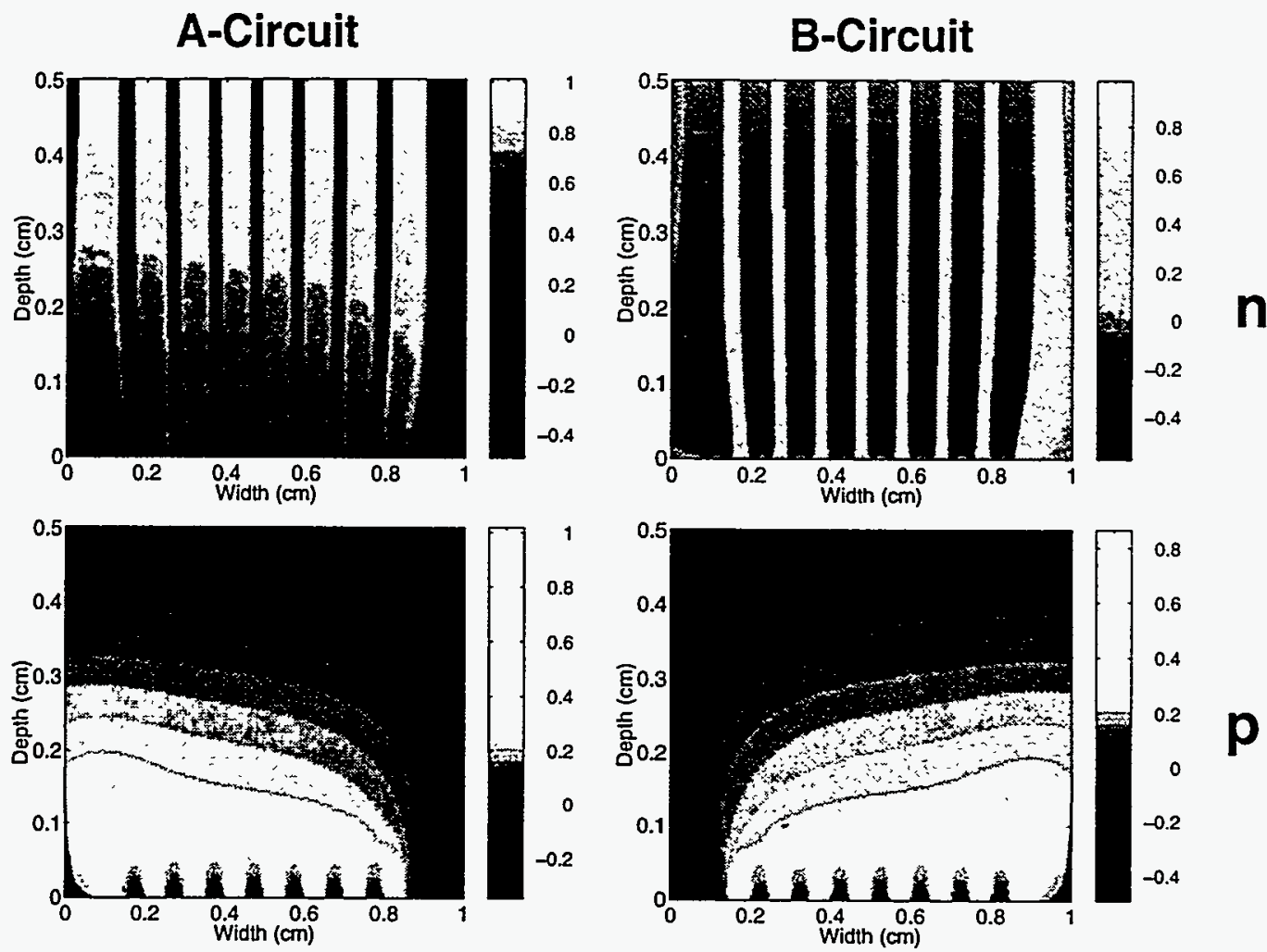

Figure 5. Induction efficiency maps for electrons (n) and holes (p) for circuits A and B of the coplanar grid detector (drift approximation).

Co-57

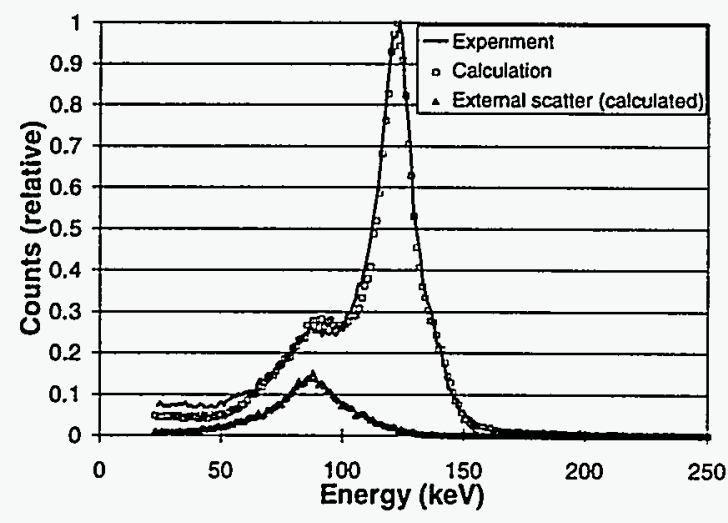

Cs-137

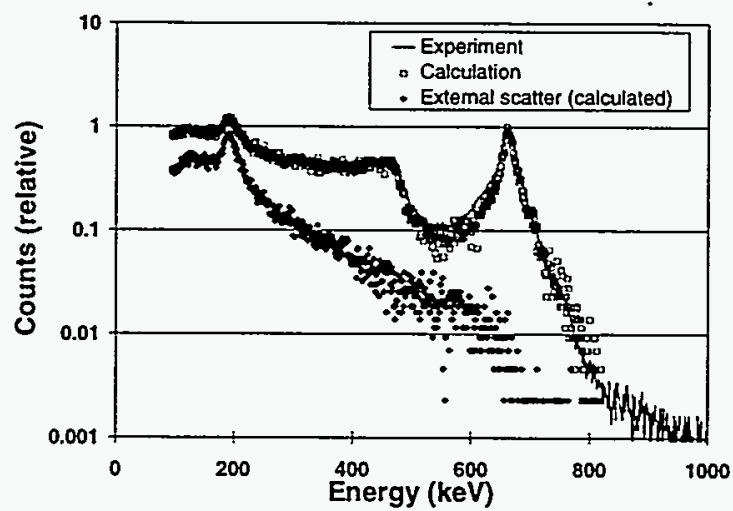

Figure 6. Experimental and calculated pulse height spectra for Co-57 and Cs-137. 


\section{DRAFT}

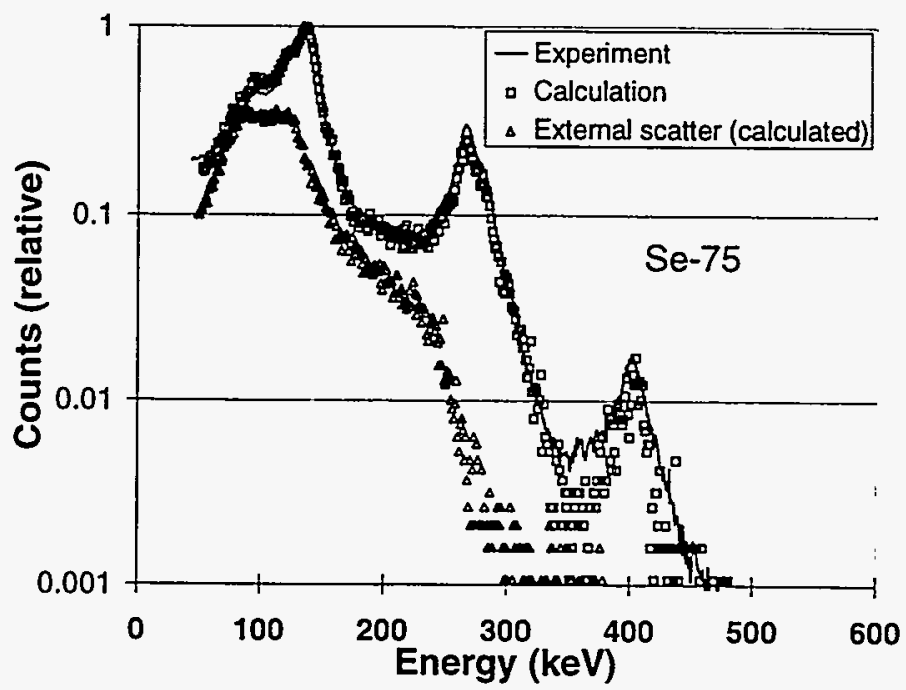

Figure 7. Experimental and calculated pulse-height spectra for a source with widely separated gamma-rays (136-, 264-, 280-, and 401-keV).

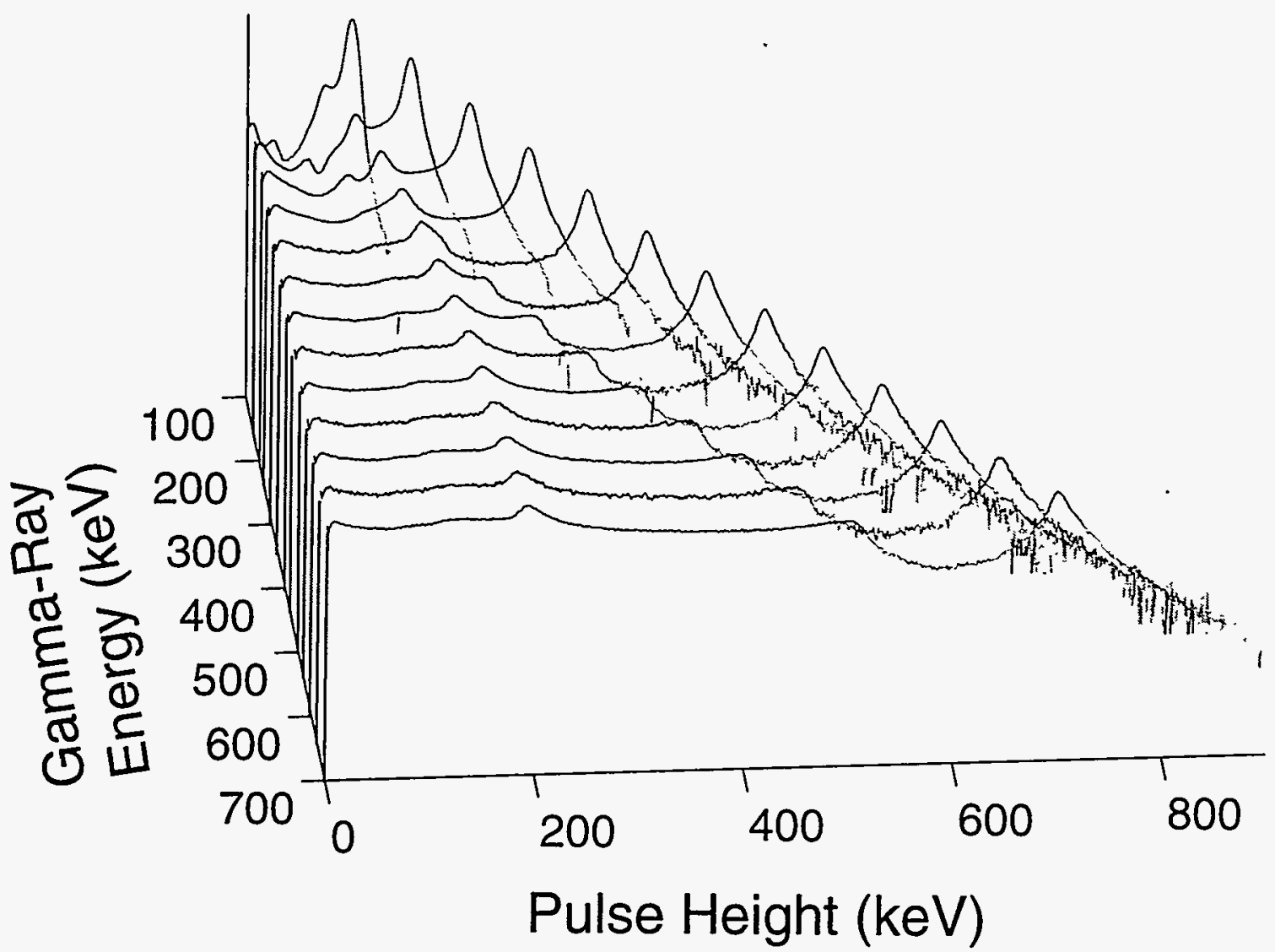

Figure 8. Response function library generated by Monte Carlo simulation for incident gamma-rays from 100 $\mathrm{keV}$ to $700 \mathrm{keV}$ in $50 \mathrm{keV}$ increments. 